\title{
A bivalent vaccine of Eimeria media and Eimeria intestinalis yields protective immunity against coccidiosis during rabbit production
}

\author{
Penny Humaidah Hamid $^{1 *}$, Widagdo Sri Nugroho $^{2}$, Sigit Prastowo ${ }^{1}$, and Rini Widayanti ${ }^{2}$ \\ ${ }^{1}$ Department of Animal Sciences, Universitas Sebelas Maret, Surakarta 57126, Indonesia \\ ${ }^{2}$ Faculty of Veterinary Medicine, Universitas Gadjah Mada, Yogyakarta 55281, Indonesia
}

\begin{abstract}
Coccidiosis is the costliest disease in the rabbit industry. The kits do not obtain maternal immunity against the diseases and therefore suckling to weaning period being the riskiest time of infection. To date, control of coccidiosis is relied on the use of chemical coccidiostat as a drug or as the substance in feed. Whilst, continuous exposure to antibiotics develops resistance and contamination in the carcass. There is no commercially provided vaccine to prevent rabbit coccidiosis cases. In this report, we processed precocious lines from the strain of Yogyakarta origin by using selection pressure to attenuate Eimeria spp. The vaccine candidate composed of $50 \%$ of each Eimeria media and Eimeria intestinalis were given orally to initiate protective immunity against rabbit coccidiosis. The vaccinated groups with $5 \times 10^{2}$ oocyst dosage exhibited $95 \%$ less total oocyst excretion compared to the non-vaccinated group. Our experiment showed no mortality and without any significantly detrimental response of vaccinated rabbits during the observation time $(P<0.0001)$. Since the oocysts in the vaccinated rabbits are excreted in dropped faecal samples, it provides recirculation and provokes a group immunity within the flock. A vaccine candidate is a promising tool as a more environmentally friendly strategy for sustainable rabbit production.
\end{abstract}

\section{Introduction}

Rabbit production in the world has been a part of efforts in fulfilling for meat consumption. Rabbits' husbandry is not only for the small family need in rural areas, but becoming a large industrial business [1]. Awareness about health and nature properties of consumed products are increasing since 2011 [2]. Some of the concern is related to development of antibiotic resistance. Bacteria affecting i. e. Staphylococcus aureus is evidently resistance to tetracycline, macrolides and multidrug, in both rabbits and farm worker [3]. Nevertheless, the use of antibiotic is unavoidably for health management in an animal production system. However, uncontrolled usage of the drugs is not only associated with meat quality and environmental issue afterwards but also high cost for farm efficiency.

\footnotetext{
*Corresponding author: pennyhumaidahhamid@staff.uns.ac.id
} 
Among the most costliest diseases in rabbit is coccidiosis [1]. The impact of the disease is not only due to the mortality of young kits but merely on subclinical forms. The surviving animals is not efficiently gaining the optimum body weight with the normal diet for fattening rabbits. The coccidiosis cases are worldwide ranging from small scale husbandry to large industry. Coccidiosis is caused by Eimeria spp. Eimeria intestinalis and Eimeria media are among the species found in prevalent. E. intestinalis is reportedly as the medium to pathogen species, whereas E. intestinalis is included in the most pathogen in rabbit [2]. These species are also found in our previous report [3] which is circulated among rabbit colonies in central part of Java, Indonesia. Although it is known that coccidiosis is threatening, the prevention of coccidiosis by using vaccine is not yet applied in the field. The farmers mainly use the sulfa-based antibiotic which is basically for avian coccidiosis.

\section{Material and methods}

Part of the experimental design regarding precocious lines was submitted and approved by ethical committee of LPPT Universitas Gadjah Mada, Indonesia. For all of the experiment we use New Zealand rabbit breed and raised in Veterinary Medicine, Universitas Gadjah Mada. The parents' rabbits and the kits were tested for coccidia free and treated with diclazuril [4]. The faecal samples were periodically observed under microscope for Eimerias. Pellets and water were provided ad libitum without chemical coccidiostat during experiment related to infection.

E. media and E. intestinalis were isolated from our work and previously published [3]. Oocyst isolation with selection pressure were generated by method previously described [5]. To analyse the reduction of pathogenic effect on reduced prepatent time, infection was performed to 3 kits with $5 \times 10^{5}$ oocysts. The kits were necropsied and the ileum of small intestine were analysed histologically.

After reduction of prepatent period obtained, each species isolated was then mixed in the same amount, for serial immunization with dosage: $5 \times 10^{2}, 1 \times 10^{3}, 2 \times 10^{3}, 4 \times 10^{3}$ and $8 \times$ $10^{3}$ oocysts orally. Challenge infection dosage was $5 \times 10^{5}$ wild type oocysts on day 14 after immunization of precocious lines. Collection of faeces started from day 3 to 12 post challenge infection and presented as the total oocyst production from experimental groups. Clinical manifestations were evaluated including lethargy, reduced appetite and fecal sample consistency. All of the data report for significance and graphical presentation were analysed by GraphPad Prism.

\section{Results and discussion}

Coccidiosis led to many consequences on rabbit husbandry. From the price due to excessive medication to long-term subclinical intestinal disorder causing lower efficiency on food conversion ratio [4]. Because the coccidia infection normally occurred in younger rabbit after around 4 weeks old, the effect is more likely due to the mortality of the younger rabbit. Instead, kits survive from the diseases frequently incapable to pass the transition from suckling to weaning. Differ from chicken coccidiosis with a well-developed vaccination for prevention [7], commercially available vaccines for rabbit coccidiosis have not available yet. Since the excessive price of medication, vaccination becomes very strategically critical to reduce infection rate and therefore maintain the most capacity of rabbit husbandry to be worthwhile.

Eimeria oocysts decreased the prepatent period E. media and E. intestinalis. Decreased pathogenicity was observed in Fig. 1 . The precocious lines were able to complete their life cycle within the host as observed during in vivo infection but less compared to normal 
Eimeria. The fecundity also obtained with gammons which led to possibility of producing more oocysts for other rabbits. However, all the complete cycle within the host do not cause severe detrimental effect within intestine and therefore rabbits showed no clinical manifestation during the pathogenicity test of precocious lines.

The oocysts of wild-type and processed for precociousness selection were our isolate previously isolated [6]. Protective immunity was observed significantly from oocyst excretions between divalent (BV)-vaccinated groups compared to control (C) when challenged with $5 \times 10^{5}$ WT (Fig. 2). Total oocysts excreted were significantly lower $(P \leq$ 0.001 ) from the vaccinated group with $4 \times 10^{2}, 8 \times 10^{2}$ and $1.6 \times 10^{3} \mathrm{BV}$ dosages. Whilst, vaccination with lower dosages i.e., $1 \times 10^{2}$ and $4 \times 10^{2}$ were not significantly reduce oocysts shedding with $P \geq 0.05$. Rabbit infected with $5 \times 10^{5}$ WT oocysts without vaccination $(\mathrm{C}$ group) showed mild diarrhoea, reduced appetite, and lethargy during 5 to 8 days postchallenge infection. Whilst, no clinical manifestations were observed in the $95 \%$ of vaccinated groups after challenge infection. The BV in our experiment also showed fecundity from the dosage of $4 \times 10^{2}$ infection. BV oocysts shedding in the environment showed enrichment ability and indicating that the attenuate-oocyst may recirculate again to infect the other rabbit further induce a flock immunity. Coccidiosis in general affects young rabbits simply after weaning (5- to 6-week-old) which are not protected by the immunity from their mother [8]. Therefore, the vaccination in around thirty days old is quite critical to prevent clinical coccidiosis and hence keeping off utilization of continuous coccidiostat in the feed and water.

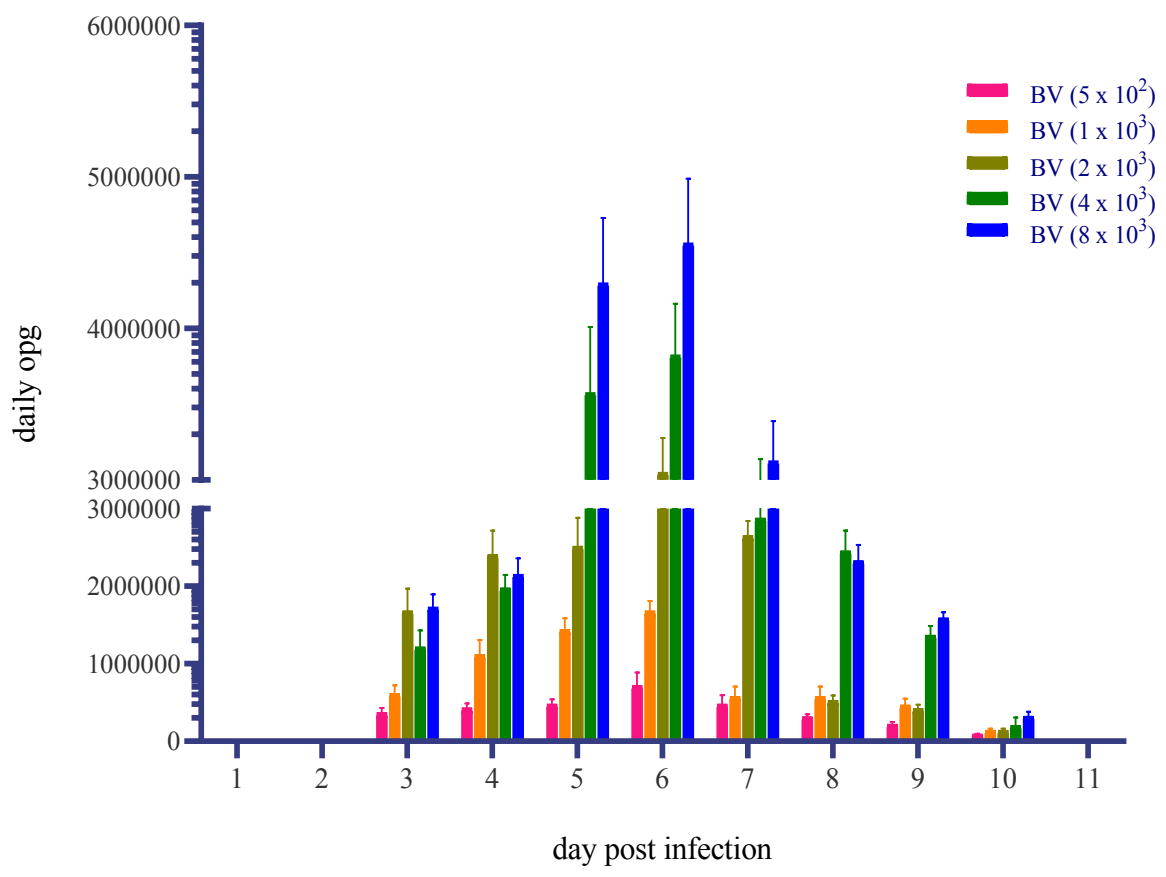

Fig. 1. Vaccination of live attenuated E. media and E. intestinalis secreted less oocysts in their faeces compared to wild-type.

The immunity against Eimeria is reportedly species specific [8]. Immunization strategy with more than one species will give benefit to obtain more complete protection in the field. This strategy was applied in the commercially available poultry coccidian [7]. Vaccination strategy with live rabbit $E$. media and E. intestinalis in this paper showed protective immunity 
against clinical coccidiosis from homolog infection. The immunity against Eimeria is reportedly species specific [8]. Immunization strategy with more than one species will give benefit to obtain more complete protection in the field. This strategy was applied in the commercially available poultry coccidian [7]. Vaccination strategy with live rabbit E. media and $E$. intestinalis in this paper showed protective immunity against clinical coccidiosis from homolog infection.

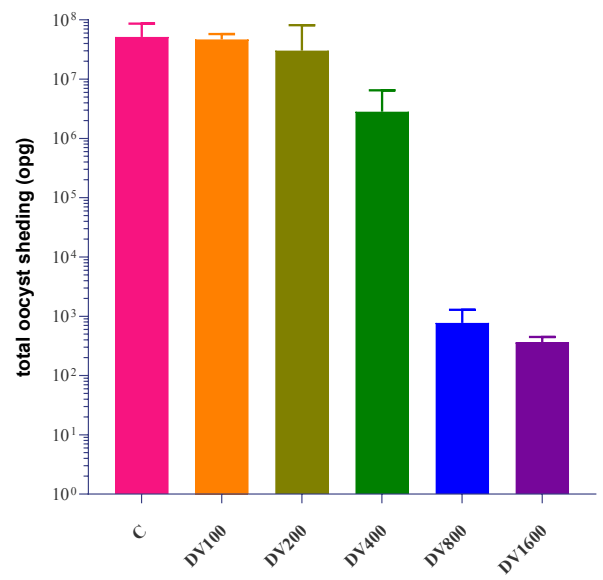

Fig. 2. Vaccination of live attenuated E. media and E. intestinalis with dosage of $4 \times 10^{2}, 8 \times 10^{2}$ and $1.6 \times 10^{3}$ were able to reduce total oocyst excretion significantly $(P \leq 0.0001)$ from the wild-type infection.

The excreted oocysts in the vaccinated rabbits may also provoke recirculation for a herd immunity if ingested by other. Protection to naturally occurred infection is difficult to be achieved during the research timeframe. Therefore, we evaluated the protection of challenge infection under laboratory condition before to ensure total numbers of oocyst administrated. Furthermore, prolonged observation time and bigger size of population test are valuable to be performed. We conclude in this report that the vaccine candidate was without detrimental effect to rabbit under field condition during the onset of coccidiosis i.e., until 14 days of infection.

\section{Conclusion}

Live-attenuated of E. media and E. intestinalis oocysts yields immune protection against coccidiosis infection. Immunity is provided by a single dosage of vaccination starting from $8 \times 10^{2}$ live attenuated of mixed oocysts. E. media and E. intestinalis may act as vaccine candidate for prevention of rabbit coccidiosis. Further studies on field trials and combination of more than 2 species can provide more information on the wide application of vaccine, its prospect and wider protective immunity.

We thank to LPDP who support and finance our work by a grant for university and industry research platform to PHH number PRJ-105/LPDP/2019.

\section{References}

1. A. Trocino, E. Cotozzolo, C. Zomeño, M. Petracci, G. Xiccato, C. Castellini, Ital. J. Anim. Sci. 18, 1361-1371 (2019)

2. R. Schleenbecker, U. Hamm, Appetite 71, 420-429 (2013) 
3. A. R. Attili, A. Bellato, P. Robino, L. Galosi, C. Papeschi, G. Rossi, E. Fileni, M. Linardi, V. Cuteri, F. Chiesa, P. Nebbia, Antibiotics 9, 673 (2020)

4. T. K. Bhat, K. P. Jithendran, N. P. Kurade, World Rabbit Sci. 4, 37-41 (1996)

5. D. W. Duszynski, L. Couch, The Biology and Identification of the Coccidia (Apicomplexa) of Rabbits of the World (Elsevier Inc., London, UK, 2013)

6. P. H. Hamid, P., S. Prastowo, Y. P. Kristianingrum, Vet. World 12, 1256-1260 (2019)

7. T.A. Ahmad, B.A. El-Sayed, L.A. El-Sayed, Trials Vaccinol. 5, 38-47 (2016)

8. M. Pakandl, Folia Parasitol. 56, 153-166 (2009) 